\title{
Des hommes en mouvement en Suisse: trois perspectives sur la masculinité
}

\author{
Hakim Ben Salah*, Jean-Martin Deslauriers* * et René Knüsel* \\ *Université de Lausanne, CH-1015 Lausanne, hakim.bensalah@unil.ch et rene.knusel@unil.ch. \\ ** Université d'Ottawa, CA- K1N 6N5 Ottawa, jean-martin.deslauriers@uottawa.ca.
}

\begin{abstract}
s
Des hommes en mouvement en Suisse: trois perspectives sur la masculinité

Cet article expose les résultats d'une démarche visant à mieux connaître la façon dont les organisations masculines suisses, de par leurs discours, se positionnent face à la redéfinition de la place et du rôle des hommes dans notre société. Après une présentation d'éléments significatifs permettant de souligner les caractéristiques récentes de la reconfiguration des rapports entre les sexes, cet article présente un survol des orientations idéologiques portées par les quelques cent organisations répertoriées. Les résultats montrent que la pluralité et la diversité des positionnements idéologiques se structurent autour de trois tendances principales, qui peuvent être analysées en fonction des transformations sociales et des changements qui ont récemment influencé les perceptions du rôle des hommes.
\end{abstract}

Mots-clés: Mouvement des hommes, Suisse, masculinités, normes de genre, organisations masculines

\section{Männer in Bewegung in der Schweiz: drei Perspektiven auf die Männlichkeit}

Dieser Artikel stellt die Ergebnisse eines Forschungsvorhabens dar, mit dem besser zu verstehen versucht wird, wie sich die Schweizer Männerorganisationen in den Diskursen bezüglich der Neudefinition der Rolle der Männer in unserer Gesellschaft positionieren. Zunächst werden einige wichtige Aspekte des jüngsten Wandels der Geschlechterrollen aufgezeigt. Der Artikel bietet sodann einen Überblick über die ideologischen Orientierungen von ungefähr hundert erfassten Organisationen. Die Untersuchung zeigt, dass sich die Vielzahl und Vielfalt der ideologischen Positionen in drei Haupttendenzen zusammenfassen lässt, die unter dem Gesichtspunkt der sozialen Transformationen und der Veränderungen, die die Rolle des Mannes in jüngster Zeit beeinflusst haben, analysiert werden.

Schlüsselwörter: Männerbewegung, Schweiz, Männlichkeiten, Gendernormen, Männerorganisationen

\section{Men on the Move in Switzerland: Three Perspectives on Masculinity}

This article presents the results of an attempt to better understand how men's organizations in Switzerland, through their discourses, view the redefinition of men's role in Swiss society. Firstly, some significant evidence concerning the recent changes in the relationships between genders is highlighted. Secondly, this article exposes an overview of the different ideological orientations of some hundred documented organizations. The results show that the plurality and diversity of ideological positions are structured around three main tendencies that can be analyzed against the background of the social transformations and changes that have recently influenced the perception of men's role.

Keywords: Men's movement, Switzerland, masculinities, gender norms, men's organizations 


\title{
Des hommes en mouvement en Suisse: trois perspectives sur la masculinité
}

\author{
Hakim Ben Salah*, Jean-Martin Deslauriers * ${ }^{*}$ et René Knüsel*
}

\section{Introduction}

Cet article présente et analyse trois perspectives sur la masculinité. Cette expression désigne un ensemble de points de vue sur la situation sociale et politique des hommes au sein d'une société (Clatterbaugh 1997). Les perspectives en question ont émergé d'une analyse de contenu des discours diffusés par des hommes en mouvement, c'est-à-dire un ensemble d'organisations réunissant des hommes consciemment impliqués dans des activités visant à (re)définir la place et le rôle de l'homme (Ben Salah 2013). L'étude présentée dans cet article - d'envergure nationale et réalisée entre 2009 et 2013 - expose les résultats issus de deux phases de récoltes de données. Les développements présentent en priorité les résultats d'une analyse de contenu réalisée sur les sites de 40 organisations jugées représentatives des principales écoles de pensée identifiées, et intègre, dans une moindre mesure, les résultats d'analyses issues d'une enquête par questionnaire diffusée auprès des membres des 103 organisations répertoriées.

Cet article présente en introduction quelques éléments significatifs permettant de relier les perspectives sur la masculinité aux changements intervenus ces dernières décennies en Suisse, en regard de la configuration des rapports sociaux entre hommes et femmes. La section méthodologique expose ensuite la façon dont les organisations masculines ont été sélectionnées et la manière dont les données ont été récoltées et analysées. La partie des résultats présente, successivement, chacune des trois perspectives sur la masculinité. Elles font ensuite l'objet d'une discussion, à la fois en référence aux éléments de contexte présentés en introduction, ainsi que sur le plan de leur potentiel d'action et de mobilisation. Enfin, la conclusion revient sur les principaux acquis de cet article.

Les regards sur la condition féminine et masculine peuvent porter aussi bien sur les plans politique, économique que culturel ou légal (Bunge 2003). Sous ses différents aspects, bien qu'encore largement à l'avantage des hommes, la situation suisse présente un bilan contrasté.

L'égalité économique entre les sexes n'est pas encore atteinte, puisqu'à qualification égale les femmes sont toujours moins bien payées que les hommes,

\footnotetext{
* Université de Lausanne, CH-1015 Lausanne, hakim.bensalah@unil.ch et rene.knusel@unil.ch.

** Université d'Ottawa, CA- K1N 6N5 Ottawa, jean-martin.deslauriers@uottawa.ca.
} 
entre $12 \%$ et $21 \%$ selon les qualifications requises pour le travail effectué (OFS 2014a). Elles sont également sous-représentées dans les positions dirigeantes sur le marché du travail (15\% de femmes en 2000) et prennent davantage en charge le fardeau des tâches domestiques et familiales, qui s'accentue encore à l'arrivée d'un enfant (OFS 2014b) ${ }^{1}$.

En politique, elles sont peu présentes au sein des hautes instances décisionnelles. En effet, en 2013, le Parlement suisse n'accueille respectivement que $31 \%$ (Conseil national) et $20 \%$ (Conseil des Etats) de femmes. En outre, les gouvernements et parlements cantonaux ne comptent qu'un quart de femmes environ - respectivement $23.7 \%$ et $25.1 \%$ (OFS 2014c).

Toutefois, malgré la persistance de certaines inégalités, la configuration des rapports entre les sexes en Suisse a subi ces dernières décennies des modifications majeures. Ainsi, un processus de rééquilibrage relatif des statuts des femmes et des hommes est engagé. La Suisse possède, par exemple, l'un des indices d'inégalité de genre $\mathrm{IGG}^{2}$ les plus faibles au monde - troisième sur 148 pays, juste derrière la Suède (Malik 2013, 168). On observe également une diminution des inégalités entre les sexes sur le plan de la formation; la proportion de la population ayant achevé une formation après l'enseignement obligatoire (formation générale ou formation professionnelle initiale) révèle une différence minime entre hommes et femmes (en 2012, $93 \%$ de femmes et $96.4 \%$ d'hommes). En outre, on recense respectivement $15.1 \%$ de femmes avec un diplôme universitaire et $13.3 \%$ d'hommes, contre $3 \%$ et $15.2 \%$ en 1991 (OFS 2014d).

Ce processus de marche vers l'égalité, certes encore contenue, est également accompagné, sur le plan légal, de l'effacement progressif des privilèges masculins. En effet, des modifications ayant trait au statut de l'homme et du père au sein de la cellule familiale et du couple marquent la disparition de privilèges. Deux changements significatifs peuvent illustrer cette évolution.

Premièrement, lors de la révision du droit matrimonial, entré en vigueur au 1er janvier 1988, l'idée d'égalité de traitement dans les effets généraux du mariage est entérinée - en ce qui concerne le nom de famille, le droit de cité et l'entretien de la famille. En outre, la notion de puissance paternelle et ses effets ont disparu, pour faire place à l'exercice conjoint de l'autorité parentale, qui concrétise une conception plus égalitaire du pouvoir parental (Reusser 2006). Ces changements dénotent, sur un plan juridique, la fin de la préséance du rôle de chef de famille, qui incombait au père jusqu'alors.

$1 \quad$ En 2013, dans un couple de partenaires avec enfant(s) (dont le plus jeune a entre 0 et 6 ans), les femmes consacrent en moyenne 56 heures hebdomadaires aux tâches domestiques et familiales, contre 31 heures pour les hommes (OFS 2014b).

2 Il s'agit d'un indice composite mesurant le déficit de progrès dans trois dimensions du développement humain dont résultent des inégalités de genre: santé reproductive (taux de mortalité maternelle, taux de fécondité des adolescentes), autonomisation et marché du travail (sièges au parlement national, population ayant suivi un enseignement secondaire ou supérieur, taux d'activité de la population active) (Malik 2013, 171). 
Deuxièmement, en 2000, le nouveau droit du divorce est entré en vigueur. Il est le reflet d'un changement général d'attitude face au divorce dans la population suisse. En effet, le taux de divorce est passé de $15.4 \%$ en 1970 à $43.2 \%$ en 2011 (OFS 2014e). Dans la loi, la procédure est allégée, puisque la notion de "faute» disparaît et il n'est plus nécessaire aujourd'hui, pour divorcer, d'apporter une preuve d'adultère ou d'abandon du domicile conjugal (Reusser 2006). Le mariage prend désormais le statut de simple contrat pouvant être dissout immédiatement par accord mutuel ou dans le respect de certains délais par une des parties.

Ces changements sont significatifs de l'allègement des contraintes juridiques qui encadrent les rapports interindividuels dans le mariage. Ils dénotent également l'affaiblissement des avantages statutaires conférés aux rôles de mari et de père.

Dans quelle mesure est-il possible d'observer l'impact de ces changements sur les points de vue sur l'identité masculine ${ }^{3}$ des hommes? Les travaux qui portent sur les discours d'hommes militants, généralement désignés par l'expression de mouvement des hommes, se sont penchés sur la question. Par des analyses des discours diffusés par des hommes impliqués dans des réflexions sur l'égalité des sexes et les identités de genre, plusieurs auteurs ont pu mettre en évidence la diversité des perspectives exprimées. Ainsi, aussi bien dans le monde anglo-saxon (Clatterbaugh 1997 ; Messner 1997; Flood et al. 2007) qu'au Québec (Lindsay et al. 2011) ou en France (Welzer-Lang 2011), plusieurs auteurs ont montré la diversité des réactions collectives des hommes aux transformations des rapports de sexe.

En Suisse, l'émergence des organisations masculines a suscité peu de recherches au sein du milieu académique. En effet, le phénomène est peu étudié ou lorsqu'il l'est, c'est à partir d'une perspective qui vise à dénoncer l'apparition de groupes dont le but serait avant tout d'attaquer les acquis des femmes (Carnal 2006; Schiess 2007).

Cette lacune a montré la nécessité d'entamer un travail de recensement et d'analyse de la diversité des positionnements des hommes en mouvement en Suisse. A partir des résultats de notre recherche, l'article cherche à comprendre de quelle manière les organisations et leurs membres, dans leurs discours, perçoivent le rôle et la place de l'homme dans la société suisse. Trois principales perspectives ont émergé de l'étude de ces organisations : défensive, expressive et relationnelle et pro-féministe. Ces trois perspectives seront successivement présentées et analysées dans cet article.

3 Les notions d'identité masculine et de masculinité sont employées comme des synonymes dans cet article. Elles désignent les rôles et attitudes jugés appropriés pour un homme dans un contexte déterminé. Cette définition repose sur une prémisse selon laquelle la masculinité est une construction sociale contingente, dont le contenu dépend de la société et de l'époque (Kimmel et Messner 1989). 


\section{Méthodologie}

\subsection{Echantillon et recrutement}

Lors d'une première étape, la réponse à notre interrogation a été fournie par des entretiens menés en 2008 auprès de 7 responsables d'organisations masculines. Les responsables ont été sélectionnés avec l'intention de contraster au mieux les différences entre les organisations, aussi bien sur le plan des actions menées (entraide, politique), de l'aire linguistique (Suisse romande, Suisse alémanique, Tessin), que des objectifs poursuivis (promotion de la paternité, introspection, combat politique, etc.).

La seconde étape a procédé à une étude des sites Internet de $40^{4}$ organisations masculines, au cours de 2009. Le même principe de maximisation des différences a été appliqué lors de la sélection des sites Internet, pour une prise en compte optimale de la diversité des discours analysés.

Enfin, la réalisation d'une enquête par questionnaire a constitué la troisième étape de cette recherche. Elle a été menée auprès des membres de l'ensemble des 103 organisations masculines répertoriées. La récolte des données a eu lieu au début 2012. Plus de la moitié des organisations visées (62) a répondu au questionnaire.

\subsection{Méthode d'analyse}

La première étape empirique a permis de rassembler un corpus de données qualitatives (transcriptions d'entrevues, pages de site Internet). Les entretiens ont été conduits de manière à obtenir des données comparables à celles récoltées sur les sites Internet des organisations. Dans les deux cas, nous avons souhaité savoir: (a) à quels problèmes l'organisation tentait de répondre; (b) quels moyens étaient employés pour atteindre les objectifs visés; (c) quelle était la situation idéale décrite par l'organisation. Par conséquent, une même analyse a pu être appliquée aux données récoltées à ces deux sources. Tout d'abord, les textes ont fait l'objet d'une lecture flottante, afin de tirer une première impression d'ensemble. Lors de cette étape, nous avons perçu les données comme un tout dont nous avons cherché à saisir le sens global (Mucchielli 2006). La lecture du corpus de données a été guidée par l'idée de repérer un ou plusieurs ensembles isomorphes.

Nous avons ensuite procédé à un repérage des premiers thèmes et sous-thèmes, afin d'obtenir un nouveau regroupement des unités textuelles (verbatim), permettant la mise en évidence d'idéaux-types. Pour chaque dimension repérée, le but de l'analyse était de faire émerger des conceptualisations constituant des ensembles cohérents et distincts. Enfin, nous avons procédé à une vérification et à un raffinement des découpages opérés, pour tester si la décomposition en catégories était

$4 \quad$ Cette étude possédant un caractère exploratoire, le répertoire des organisations a évolué au gré des découvertes. Le nombre de 40 organisations représentait la moitié des sites Internet identifiés au moment de la première récolte empirique. Cet échantillon a permis d'atteindre la saturation des données. 
suffisamment robuste pour intégrer les données d'autres textes et si les catégories étaient mutuellement exclusives.

La seconde étape a comporté une récolte de données quantitatives. Les analyses statistiques réalisées sont variées (ANOVA, analyses factorielles, tableaux croisés, fréquences). Pour cet article, la priorité étant donnée aux résultats de la phase qualitative, nous ne mobilisons que les résultats d'analyses de fréquences et les données issues des tableaux croisés documentant les caractéristiques sociodémographiques des membres des organisations masculines.

\section{Résultats}

\subsection{Résultat de la catégorisation: trois perspectives sur la masculinité}

Cette section propose une présentation synthétique des trois perspectives sur la masculinité identifiées dans les discours des organisations masculines et de leurs responsables. Cette présentation est structurée selon les catégories émergées suite à l'analyse de contenu décrite au point précédent. Elles permettent une différenciation systématique des points de vue exprimés. Ces catégories sont à la fois originales issues d'une démarche inductive - et inspirées des travaux réalisés dans d'autres contextes (Clatterbaugh 1997; Messner 1997).

Ainsi, les discours analysés concernent: (a) des constats sur la masculinité; (b) un idéal de masculinité à atteindre. Ces deux catégories génériques se déclinent toutes deux selon les dimensions décrites ci-après.

Dimension sociale et légale. Cette dimension regroupe les éléments décrivant la position ou les places respectives des hommes et des femmes au sein de la société dans son ensemble ou au sein d'une des deux sphères de la société, divisées selon la dichotomie public/privé. Elle inclut principalement la politique et le travail rémunéré/la sphère domestique. De plus, les propositions qui font référence aux lois ou qui définissent le statut des hommes et des femmes sur les plans familial, politique et économique de notre société sont incluses.

Rapport à l'autre. Cette dimension fait référence aux relations interindividuelles. Elle inclut les propositions caractérisant les relations entre individus (femmeshommes, hommes-hommes).

Dimension psychologique et axiologique. Cette dimension comprend des propositions portant sur les pensées, les attitudes, les sentiments, les croyances ou les valeurs. Elles décrivent également la façon qu'ont les hommes et les femmes de gérer leur intériorité.

Les sections suivantes exposent le contenu des trois perspectives sur la masculinité. Les catégories discursives principales (constats sur la masculinité et idéal de masculinité) vont être étudiées dans les trois perspectives. Le contenu est également 
décrit pour chacune des sous-catégories (Dimension sociale et légale, Rapport à l'autre, Dimension psychologique et axiologique).

\subsubsection{La perspective défensive}

La perspective défensive reflète le point de vue d'une majorité d'organisations (58\%). Il s'agit principalement d'organisations paternelles (Coordination romande des organisations paternelles - CROP, Interessengemeinschaft geschiedener und getrennt lebender Männer - IGM Schweiz), d'organisations politiques de défense des droits des hommes (Männer und Gleichberichtigung) et d'associations antiféministes (Antifeminismus.ch). L'enquête réalisée auprès des membres a révélé qu'une majorité des hommes appartenant à ce type d'organisations $(\mathrm{N}=136)$ est de niveau universitaire ${ }^{5}$ $(62 \%)$. De plus, ces hommes possèdent un statut socio-professionnel ${ }^{6}$ diversifié, $^{2}$ comprenant près d'un tiers de dirigeants et gérants (29\%), un quart d'hommes exerçant une profession intermédiaire $(25 \%)$ et une proportion équivalente de personnes ayant une profession intellectuelle ou scientifique $(24 \%)$.

La partie suivante expose la catégorie des constats sur la masculinité dans la société suisse, selon la perspective défensive.

\section{Constats sur la masculinité dans la société suisse selon une perspective défensive}

Dimension sociale et légale. Sur le plan de la position sociale et légale, les organisations défensives sont très présentes, puisqu'une très grande majorité $(90 \%)$ exprime une opinion sur une quinzaine de thématiques (santé des hommes, service militaire obligatoire, divorce, rentes sociales, quotas, éducation, etc.). Les organisations défensives recourent à un certain nombre de stratégies discursives, qui vont de l'opposition frontale au renversement du discours d'organisations féministes ou autres institutions officielles garantes de la réalisation de l'égalité entre les sexes (Bureaux de l'égalité).

Premièrement, les organisations défensives développent un contre-discours à travers des arguments qui visent à atténuer, voire à nier, les inégalités avérées entre les sexes. Ainsi, les écarts salariaux entre hommes et femmes sont minimisés ou niés (“pour un travail équivalent, le salaire est le même» (IGM Schweiz 2009)7). Le temps consacré aux tâches ménagères et familiales est jugé équivalent ("les tâches consacrées à la famille sont à peu près égales entre le père et la mère» (CROP 2009a).

Deuxièmement, ces organisations opèrent un renversement de l'argumentation développée par certains discours d'obédience féministe. Par exemple, en réponse à l'affirmation que les femmes au foyer sont exploitées par leur conjoint parce qu'elles effectuent un travail domestique gratuit, ces organisations rétorquent que leur absence

5 Ecole polytechnique fédérale, université et haute école spécialisée.

$6 \quad$ Les catégories utilisées sont celles de la classification internationale ISCO-88.

$7 \quad$ Dans les parties 3.1.1 à 3.1.3, l'utilisation de guillemets signale des passages tirés soit de la documentation provenant des sites Internet des organisations, soit du corpus d'entretiens menés avec des responsables. 
sur le marché du travail est, au contraire, à l'avantage des femmes. Elle leur offrirait l'opportunité de se réaliser, tout en profitant du salaire de leur mari.

Troisièmement, les organisations défensives affirment que les hommes sont victimes d'injustices dans un certain nombre de domaines. Le service militaire obligatoire et ses substituts (service civil, protection civile et taxe d'exemption) ne s'appliquent qu'aux hommes; le versement d'un nombre de rentes vieillesse (AVS) plus important aux femmes en raison d'une espérance de vie supérieure à celle des hommes ou encore l'attribution automatique de l'autorité parentale à la mère dans les cas où les couples ne sont pas mariés, représenteraient autant de situations iniques. Ainsi, sur l'ensemble de ces questions et sur un certain nombre d'autres encore (quotas et mesures d'encouragement réservées aux femmes dans le monde du travail, adoption du langage épicène, etc.), les organisations défensives dénoncent le non-respect de la mise en application du principe d'égalité de traitement entre hommes et femmes inscrit dans la Constitution suisse.

Rapport à l'autre. Sur ce point, les organisations défensives développent, de façon majoritaire ( $80 \%$ ), les thèses suivantes: (a) les hommes et les femmes adoptent des comportements négatifs dans des proportions égales (violence, harcèlement sexuel ou moral, etc.); (b) dans l'opinion commune et les médias, les comportements négatifs commis par les hommes sont exagérés; (c) en revanche, ceux perpétrés par les femmes sont minimisés, voire passés sous silence.

Pour illustrer la première des trois thèses, deux extraits sont reproduits ici, qui concernent les tâches domestiques et familiales, ainsi que la violence parentale:

(...) des statistiques donnent de forts indices que la violence physique envers les enfants serait perpétrée à environ $50 \%$ par les mères et $50 \%$ par les pères (...). La charge totale d'activités consacrées à la famille est à peu près égale entre les mères et les pères. (CROP 2009a)

On trouve, de plus, l'idée que la société helvétique tendrait vers une «survalorisation des compétences et des comportements féminins" (Boys to Men 2009), chez les jeunes garçons. Cela, au détriment de comportements perçus comme «traditionnellement masculins» (Boys to Men 2009) subissant, selon cette ligne de pensée, une tendance générale à la dévalorisation. Il s'agit, notamment, du fait de "se mesurer» (Boys to Men 2009), aussi bien physiquement qu'intellectuellement, entre hommes, d'entretenir les rivalités masculines, mais également de faire preuve de courage.

Psychologique et axiologique. Sur cet axe, les associations paternelles décrivent les pères divorcés ou séparés comme des êtres en souffrance, qui expérimentent «une augmentation des difficultés psychiques (...) et du risque de suicide » (CROP 2009b) due au manque de reconnaissance de leurs droits. Cet état s'expliquerait notamment par la frustration de certains pères désireux de remplir leur rôle paternel auprès de leurs enfants après une séparation. Des pères qui seraient confrontés à l'impossibi- 
lité de maintenir une relation avec leurs enfants, en raison d'entraves créées par le système judiciaire ou le comportement de certaines mères.

En outre, ces organisations mettent en évidence l'absence aussi bien physique que symbolique des pères, en particulier dans le domaine de l'éducation, que ce soit dans un cadre familial, privé ou dans le domaine de l'enseignement primaire public. En outre, l'absence du père est mentionnée comme une cause première de déséquilibre psychique, chez les enfants en général et chez les jeunes hommes en particulier. L'instabilité identitaire des hommes serait également causée par le fait d'être tiraillés par les injonctions paradoxales des femmes: se montrer «doux et compréhensif» (Macho Man 2009) ou, au contraire, incarner l'«image virile du macho» (Macho Man 2009).

\section{Idéal de masculinité à atteindre dans la société selon une perspective défensive}

Cette section décrit l'idéal de masculinité selon la perspective défensive, qui se décline également d'après la structure tridimensionnelle exposée auparavant dans cet article.

Dimension sociale et légale. Les organisations défensives, qui mettent l'accent sur les inégalités sociales dont les hommes sont victimes, formulent logiquement des revendications visant à redresser les torts qui leur sont causés: suppression du service militaire obligatoire et de la taxe d'exemption pour les hommes; instauration de l'autorité parentale conjointe automatique et mesures d'égalisation des statuts juridiques du père et de la mère; propositions visant à protéger les hommes d'abus (violence, fausses accusations) de la part des femmes dans le cadre conjugal et lors de divorces. En outre, les organisations alignées sur cette perspective se positionnent contre les mesures politiques de quotas visant à favoriser l'accès des femmes aux positions de pouvoir, que ce soit en politique ou dans la sphère du travail rémunéré.

Rapport à l'autre. Sur cette dimension, les organisations défensives déplorent une mise en danger de l'idéal masculin. Elle serait causée, entre autres, par une mainmise des femmes sur l'éducation, d'une part, et, d'autre part, par une féminisation de la société à travers la valorisation des compétences féminines au détriment des aptitudes masculines. En conséquence, pour ces organisations, les comportements doivent s'ancrer avant tout dans la différence avec la féminité, qui passe par la préservation et la revalorisation d'attitudes décrites comme typiquement masculines: s'exprimer bruyamment, faire preuve de courage et savoir prendre des risques, se montrer affirmé. On suggère également de préserver et d'encourager les liens de sociabilité entre hommes lors d'occasions spécifiques: match de football, karting.

Psychologique et axiologique. Contrairement aux deux autres tendances qui abordent plus amplement cette dimension, moins d'éléments ressortent dans cette perspective - $20 \%$ des organisations défensives s'y situent. Toutefois, quelques occurrences traduisent une volonté de valoriser auprès des hommes une attitude de retenue face à ses propres émotions. Une certaine forme de stoïcisme, pourrait-on également dire, qui fait partie des attributs d'une masculinité traditionnelle. 


\subsubsection{La perspective expressive et relationnelle}

Un deuxième groupe d'organisations (37\%) prône une masculinité relationnelle et expressive. Cette appellation fait référence à un ensemble de valeurs prédominantes dans l'idéal de masculinité porté par cette perspective. Il s'agit des valeurs d'expression de soi, caractéristiques du passage des sociétés occidentales à la postmodernité (Inglehart et Welzel 2005). Par ailleurs, elle évoque également la prépondérance de la valorisation des aspects relationnels au sein des modèles prônés. Les organisations qui adoptent cette perspective ont des visées diverses - lutte contre la violence conjugale (services aux hommes auteurs de violence), travail de groupe sur la masculinité d'inspiration psychanalytique, action politique.

Notre enquête permet également de souligner quelques caractéristiques des hommes adhérant à cette perspective $(\mathrm{N}=74)$. Une majorité de membres $(88 \%)$ possède un statut socio-professionnel très élevé, de dirigeant ou de gérant ${ }^{8}(23 \%)$ ou exerce une profession intellectuelle ou scientifique (55\%). Le niveau de formation de ces hommes est également très élevé, puisque plus de trois quarts (83\%) d'entre eux déclarent être titulaires d'un diplôme d'une université ou d'une haute école spécialisée.

La partie suivante expose donc le positionnement de la perspective expressive et relationnelle, en commençant par la catégorie des constats sur la masculinité.

\section{Constats sur la masculinité dans la société suisse selon une perspective expressive et relationnelle}

Dimension sociale et légale. Dans cette perspective, les inégalités envers les femmes sont reconnues. En effet, les hommes sont décrits comme occupant une position privilégiée par rapport aux femmes dans les sphères politique et professionnelle. Toutefois, l'explication de ce fait correspond ici à un renversement de la rhétorique féministe. Contrairement à cette dernière - qui voit dans ce déséquilibre la marque d'une domination exercée par les hommes sur les femmes - l'argumentation développée par cette perspective est davantage focalisée sur les contraintes subies par les hommes. La masculinité traditionnelle serait soutenue par des normes sociales et juridiques contraignantes pour les hommes. Dans le premier cas, au sein du monde du travail, le faible recours au travail à temps partiel serait conditionné par de forts préjugés négatifs pesant sur les hommes désirant réduire leur temps de travail. Dans le second, certaines obligations légales, à l'instar de l'obligation de servir dans l'armée, agiraient comme des contraintes, ayant pour effet, par exemple, de limiter l'engagement des hommes auprès de leur famille.

Rapport à l'autre. Sur ce point, la critique porte sur l'isolement affectif et physique qui imprègne les rapports entre hommes. Ces derniers sont décrits comme

8 Dans ce groupe sont classées les personnes qui assument la gestion d'une entreprise ou, le cas échéant, d'un organisme, pour leur propre compte ou pour le compte de son propriétaire avec le concours d'un seul autre cadre de direction et d'assistants subalternes (cf. BIT n. d.). 
avares de démonstrations d'affection et retranchés dans des attitudes stéréotypées et manquant d'authenticité. En effet, les hommes seraient liés par la peur. Peur, d'une part, de l'homosexualité ou, d'autre part, crainte d'être assimilé aux femmes ou au féminin.

En outre, la dépendance affective de l'homme envers sa compagne caractériserait les rapports entre hommes et femmes dans le couple. La plupart des hommes se trouveraient dans une situation de report affectif, surinvestissant la dimension émotionnelle de la relation avec leur compagne, en raison précisément de la pauvreté des relations entre hommes.

Psychologique et axiologique. Le modèle traditionnel masculin est ici perçu comme défaillant; les hommes, conditionnés par un processus de socialisation et d'éducation les poussant à adhérer à un modèle de masculinité archaïque, intérioriseraient une injonction "à ne pas ressentir, ni exprimer leurs sentiments", à " refouler leur sensibilité », «de peur de perdre la face» (Réseau hommes romand 2009). Ainsi, en raison d'une pression continue à se montrer forts, les hommes sont décrits comme démunis face à certaines émotions, comme la tristesse ou le sentiment d'impuissance. Cette crainte induirait des répercussions sur le plan relationnel, comme le discours sur la dimension précédente le montre. L'intériorisation de «l'obligation de se montrer performant» aurait des répercussions sociales. En effet, en raison de la pression intérieure causée par cette norme, les hommes adopteraient des comportements préjudiciables, voire nocifs, pour eux comme pour leur entourage: surinvestissement dans le travail, comportements à risque, consommation de drogues et d'alcool, etc. Certaines explications de cet état de fait sont fournies par des théories psychanalytiques attribuant cette déficience à l'absence de modèles masculins "sensibles» dans l'entourage des hommes, cause d'une incapacité à manifester leurs émotions (Corneau 1989) comme le fait de pleurer. Ainsi généralement, le jeune homme, privé au cours de son éducation de modèle paternel sensible, n'accède pas à la possibilité d'exprimer ses sentiments et de se montrer vulnérable.

La section suivante aborde le positionnement de la perspective expressive et relationnelle sur l'idéal de masculinité à atteindre dans la société.

\section{Idéal de masculinité à atteindre dans la société selon une perspective expressive et relationnelle}

Dimension sociale et légale. La majorité des organisations adoptant cette perspective se prononcent peu sur cette dimension. Cela peut s'expliquer par le fait que la majorité d'entre elles sont des organisations d'entraide. En réponse au déficit constaté dans la gestion des émotions, elles privilégient une action tournée vers l'exploration de l'intime (groupes de parole, danse) et non vers la réflexion, ni l'action politique.

Cependant, une organisation en particulier (Maenner.ch) développe des activités sur le plan politique. En réaction aux constats de comportements masculins stéréotypés, l'objectif est de réformer les structures sociales (lois, pratiques, men- 
talités) traditionnelles. Les actions privilégiées pour mettre en œuvre cette réforme structurelle sont la participation à des activités parlementaires, la formulation de propositions de réformes légales concrètes ou le développement de campagnes de sensibilisation, par exemple en incitant les cadres à réduire leur temps de travail. Le point commun de ces actions est de créer des conditions cadre permettant aux hommes de s'extraire des carcans de la masculinité traditionnelle et de bénéficier de modèles d'affirmation plus libres et égalitaires (accès au temps partiel, meilleure implication au sein de la sphère domestique, etc.).

Rapport à l'autre. Pour une majorité d'organisations de cette obédience (60\%), les hommes devraient, dans ce domaine, développer un certain nombre de compétences. Notamment, des capacités d'écoute et de communication avec leur entourage, qu'il soit masculin ou féminin. En ce qui concerne les rapports entre hommes, en réponse à l'isolement relationnel pointé dans les constats, les propositions portent globalement sur l'instauration d'un dialogue se basant sur l'" expression du ressenti», du "vécu personnel»: "parler en je», sur "ce que ça me fait plutôt que sur ce que j'en pense", "ne pas juger ce qui vient» (Réseau hommes romand 2009), etc. L'échange d'expériences, de connaissances, la prise en compte de l'avis et de la parole de l'autre sont également encouragés.

Psychologique et axiologique. Sur un plan psychologique, ce sont avant tout les capacités d'introspection qui sont valorisées (60\% des organisations). Pour les organisations qui travaillent sur les questions de violence conjugale, en particulier, il faut d'abord appliquer ces procédés à sa propre situation. L'auto-observation constitue l'outil nécessaire pouvant mener à la prise de conscience et à l'acceptation de ses états intérieurs, notamment de ses vulnérabilités, dans un premier temps, puis au dépassement des comportements violents.

\subsubsection{Perspective pro-féministe}

Un dernier groupe d'organisations (moins de $5 \%$ ) adhèrent à un modèle de masculinité pro-féministe. Elles réunissent des hommes proposant une critique forte de la masculinité traditionnelle, perçue comme une construction sociale à la fois productrice et reproductrice d'inégalités. Les hommes soutenant ce point de vue sont principalement réunis en groupes de parole. Cette perspective semble entrée dans une phase de déclin, puisque les deux seules organisations ayant adopté ce point de vue ont été dissoutes durant le temps de la recherche. De ce fait, notre enquête n'a pas permis d'apporter de renseignements sur les caractéristiques sociodémographiques des hommes ayant adhéré à cette perspective.

\section{Constats sur la masculinité dans la société suisse selon une perspective proféministe}

Dimension sociale et légale. Cette perspective se concentre sur les inégalités sociales qui pèsent sur les femmes. La place des hommes est perçue comme globalement avantageuse par rapport à celle des femmes, et ce dans de nombreux domaines. 
Dans la sphère privée, cette domination est perceptible par l'assignation des tâches domestiques aux femmes et la faible implication des hommes dans ces activités socialement peu valorisées. De plus, la domination dans la sphère privée est doublée d'une domination au sein de la sphère professionnelle - majorité d'hommes aux postes de cadres, salaire des hommes en moyenne plus élevé que celui des femmes et politique - majorité d'hommes parmi les élus et responsables des parlements (cantonaux et fédéral). Contrairement à la perspective précédente, la reconnaissance d'inégalités envers les hommes sur certaines questions (divorce, obligation militaire, etc.) est absente.

Rapport à l'autre. Sur ce plan, cette perspective propose une critique calquée sur le féminisme radical. Les hommes sont avant tout perçus comme imposant des rapports de domination et de violence à autrui, vis-à-vis des femmes en particulier, à l'égard desquelles ils adopteraient des comportements de prédateurs sexuels. En outre, selon ce point de vue, les hommes seraient tous «à des degrés divers, auteurs de violence domestique, aussi bien physique en portant des coups, que morale, par des menaces et l'usage de violences verbales». Entre hommes, les rapports sont principalement décrits comme marqués par la rivalité et la méfiance.

Psychologique et axiologique. Le propos, sur ce point, consiste en une critique de trois traits principaux caractérisant les valeurs masculines, globalement empreintes de la volonté de dominer autrui. A l'encontre des femmes, c'est la misogynie, correspondant à un sentiment de mépris, qui prédomine. Entre hommes, c'est l'homophobie qui transparaît avant tout. Elle est un sentiment qui permet de se démarquer du féminin, par peur d'y être assimilé. De plus, face au féminisme, c'est la résistance psychologique et le refus des critiques qui caractériseraient au mieux les valeurs masculines.

Enfin, les hommes qui adhèrent à une perspective pro-féministe accordent peu d'importance, quand ils ne la nient pas, à l'expression de la souffrance masculine, qualifiée de "jérémiades». En effet, la seule souffrance masculine tolérée est celle découlant du malaise d'appartenir à la classe oppressive des hommes.

\section{Idéal de masculinité à atteindre dans la société selon une perspective proféministe}

Cette section décrit la perspective pro-féministe sur l'idéal de masculinité.

Dimension sociale et légale. En réponse au constat d'inégalités frappant les femmes, cette perspective soutient un agenda de quotas favorisant la présence de femmes dans les domaines traditionnellement dévolus aux hommes (politique, universités, postes de direction, etc.). En outre, certaines organisations préconisent auprès de leurs membres une mise en pratique concrète de leur adhésion de principe. Premièrement, par un retrait volontaire du monde du travail à travers une diminution du taux d'activité, jusqu'à hauteur de $50 \%$. Deuxièmement, les hommes sont incités à s'investir autant que leur conjointe dans les activités domestiques non 
rémunérées (tâches ménagères, soins aux enfants), afin de favoriser concrètement l'accès des femmes au monde du travail.

Rapport à l'autre. Sur ce point, la perspective pro-féministe encourage les hommes à délaisser toute forme de violence, en priorité vis-à-vis des femmes. Une résistance active est prônée face aux comportements machistes et misogynes. Par exemple, on encourage à manifester publiquement son opposition verbale ou son désaccord vis-à-vis des attitudes sexistes des hommes. Au cœur de cette transformation, les hommes sont incités à réduire l'écart entre l'adhésion à une idéologie égalitaire et leurs comportements concrets, quotidiens.

Psychologique et axiologique. Sur cette dimension, c'est également une attitude d'autocritique qui est encouragée: se reconnaître soi-même auteur de comportements violents et de l'exercice de la domination, prendre conscience du fait de bénéficier, en tant qu'homme, de nombreux privilèges sociaux au détriment des femmes. L'adhésion personnelle à l'analyse féministe radicale des rapports sociaux de sexe et la reconnaissance de l'existence d'une société patriarcale est également valorisée.

\section{Discussion}

Les trois perspectives identifiées révèlent une forte polarisation des opinions parmi les membres d'organisations masculines sur des aspects aussi bien politiques, psychologiques que philosophiques. Ce constat rejoint les observations menées dans d'autres contextes, au Québec (Lindsay et al. 2011), aux Etats-Unis (Clatterbaugh 1997 ; Messner 1997) et en Australie (Karoski 2007). En effet, la Suisse abrite deux pôles très opposés, la perspective pro-féministe et la perspective défensive, présentant de fortes similitudes avec les tendances Men's rights et Profeminist men (Etats-Unis). En Suisse également, la potentialité d'actions et de réflexions communes de ces organisations se heurte à de fortes incompatibilités idéologiques. Ce résultat suggère la présence d'un ensemble hétérogène d'hommes préoccupés par différents aspects des réalités masculines plutôt qu'un mouvement social, unifié autour d'une vision ou d'objectifs communs. Ce résultat permet donc de nuancer les conclusions de travaux antérieurs sur la Suisse, qui concluaient presque unanimement à la seule présence d'un mouvement de ressac.

Concernant l'envergure du phénomène des hommes en mouvement, les estimations existantes pour la Suisse, qui font état de quelque 10'000 membres d'organismes masculins (Theunert 2012), reposent sur des calculs trop imprécis pour être considérés comme des acquis. De plus, cette enquête, qui n'avait pas pour objectif de réaliser un recensement du nombre de membres des organisations, n'a pas généré de résultats précis sur ce point, et mériterait d'être davantage approfondie.

Par-delà les considérations portant sur l'ensemble du mouvement, les trois soussections suivantes proposent des analyses centrées sur chacune des trois perspectives. 
La perspective défensive exprime une position en décalage avec le constat des inégalités entre les sexes en Suisse, notamment en ce qui concerne la situation des hommes et des femmes en politique et dans le monde du travail. En effet, par de nombreux aspects - plus particulièrement sur la dimension sociale et légale - la vision défendue par cette école de pensée décrit un retournement de situation où les hommes seraient désormais les principales victimes d'inégalités dans de nombreux domaines (divorce, armée, travail, etc.). Malgré ce positionnement à contre-courant, elle semble être la perspective qui récolte le plus d'adhésion, ne serait-ce qu'au travers de la présence marquée des nombreuses organisations paternelles, seules à être présentes dans les trois régions linguistiques.

L'une des interprétations possibles est de voir dans le contre-pied pris par ces organisations le signe de la présence d'un mouvement revanchard envers les femmes. Ainsi, leur but serait de bloquer ou de restreindre leur accès à des droits et des conditions de vie équivalentes à celles des hommes. Dans d'autres contextes (Etats-Unis, Canada), c'est généralement l'interprétation privilégiée par les recherches féministes ou pro-féministes de ce courant de pensée (Dufresne 1998; Lingard et Douglas 1999; Thiers-Vidal 2002; Dupuis-Déri 2004; Blais et Dupuis-Déri 2008). En Suisse, le fait de souligner la présence d'inégalités touchant les hommes a été interprété jusqu'ici comme une stratégie de détournement de l'attention publique sur les hommes, dans le but d'entraver une "véritable» réalisation de l'égalité qui concernerait uniquement la situation des femmes (Schiess 2007).

Ce phénomène peut effectivement être assimilé, dans son expression la plus radicale, à un mouvement de ressac. Cependant, il serait risqué de réduire cette tendance à cette seule interprétation. Le danger est de passer sous silence l'existence de problèmes sociaux touchant effectivement les hommes. La problématique de l'autorité parentale conjointe, pour laquelle les organisations paternelles ont lutté depuis de nombreuses années en Suisse, est révélatrice à cet égard. Si l'on prend en considération les cas où l'autorité parentale est attribuée à l'un des deux parents uniquement, entre 2000 et 2010, 8636 femmes, en moyenne, obtiennent l'autorité parentale contre 728 hommes, c'est-à-dire plus de dix fois moins ${ }^{9}$. Au-delà des causes qui entraînent cette différence, notons que le retrait de l'autorité parentale équivaut, selon le droit suisse, à priver le parent de son rôle de représentant et d'éducateur de l'enfant et crée un déséquilibre des pouvoirs entre les ex-conjoints:

(...) le fait que l'autorité parentale est octroyée le plus souvent à la mère donne une position forte à celle-ci pour négocier son accord à l'autorité parentale conjointe, étant donné qu'elle peut faire dépendre cet accord de l'obtention d'avantages sur d'autres points; elle peut par exemple demander une pension alimentaire plus élevée contre son accord à une autorité parentale conjointe.

9 Moyennes réalisées à partir des données de la statistique suisse (OFS 2014f). 


\section{Par ailleurs, elle peut refuser son accord sans motifs. Et à défaut d'un accord, l'autorité parentale conjointe ne peut pas être imposée. (DFJP 2009)}

Les organisations paternelles, qui ont pris une part active dans la lutte pour la réforme de l'autorité parentale, qu'ils souhaitaient voir attribuer d'office aux deux parents, réunissent bon nombre d'hommes qui avaient l'impression d'être discriminés à cet égard ${ }^{10}$. Cette situation de frustration permet d'expliquer la présence d'un discours qui accentue les inégalités vécues par les hommes dans une partie des organisations étudiées. Par ailleurs, le fait que cette iniquité soit désormais reconnue officiellement permet de questionner la thèse de l'instrumentalisation de cette question par des hommes uniquement motivés par une volonté de nuire à leurs ex-conjointes et d'entraver leurs droits.

Nos constats tendent, au contraire, à souligner qu'à certains égards, la tendance défensive dénote la présence d'un mouvement cherchant non pas à priver les femmes de leurs droits, mais plutôt à rectifier et à égaliser les statuts des hommes dans certains domaines, où la marche vers l'égalité des femmes mérite d'être accompagnée de réformes attentives à la situation de ces derniers.

De plus, les revendications de certaines organisations défensives (paternelles) dénotent un mouvement d'adaptation aux reconfigurations des rapports entre les sexes. Plus précisément, le remaniement du statut de la parentalité proposé par les organisations paternelles peut être interprété comme une réaction à la diversification des configurations familiales actuelles. En effet, l'augmentation du nombre de divorces et des familles recomposées favorise l'apparition dans les rapports éducatifs de figures parentales alternatives (nouvelle compagne ou nouveau compagnon du parent séparé). Cette évolution est susceptible, dans certains cas, de conduire à une fragilisation potentielle, voire une rupture du lien de filiation originel. En réaction à cette évolution, la perspective défensive émet la volonté de faire reconnaître la paternité et la parentalité indépendamment du lien conjugal. Par exemple, la revendication d'attribuer l'autorité parentale conjointe d'office apparaît comme une volonté d'autonomiser et de protéger le lien de filiation, quel que soit l'état des rapports parentaux. Par cette volonté d'insérer les rapports de filiation dans de nouveaux cadres légaux, la perspective défensive renferme une tentative collective d'adaptation à la diversification des formes familiales et des styles de vie.

Enfin, on peut s'interroger sur les potentialités futures de mobilisation de la perspective défensive. Bien qu'elle soit aujourd'hui la plus présente, du moins en termes d'organisations, ses potentialités d'expansion paraissent limitées. En effet, l'autorité parentale conjointe étant désormais la règle, l'une des revendications majeures des organisations paternelles a abouti. Or, comme la recherche sur les mouvements sociaux le montre (Miller 1999), la démobilisation, voire la dissolution, sont des

10 Le Conseil fédéral a récemment fixé l'entrée en vigueur des dispositions d'exécution relatives à l'autorité parentale conjointe au 1er juillet 2014, respectivement au 1er janvier 2015. 
effets potentiels qui guettent les organisations des mouvements sociaux au moment où celles-ci atteignent tout ou partie des objectifs politiques qu'elles poursuivaient.

La perspective expressive et relationnelle présente, à priori, plusieurs caractéristiques intéressantes en termes de potentiel de mobilisation. Tout d'abord, elle intègre potentiellement un large spectre idéologique en proposant une conciliation de positions généralement placées en opposition; elle prône à la fois la prise en considération de la souffrance des hommes, tout en maintenant une ouverture envers l'analyse féministe, dont elle soutient l'objectif d'amélioration de la situation sociale des femmes. Ce positionnement ne se retrouve pas de façon explicite dans d'autres contextes déjà étudiés (Etats-Unis; Québec; France; Australie). En outre, au cœur de cette perspective, on retrouve un ensemble de valeurs fortes que ses tenants associent à la masculinité - liberté de choix, importance du relationnel dans les modèles éducatifs et valorisation de l'expression des émotions. Ces valeurs coïncident fortement avec les tendances majoritaires observées au sein de la population suisse, qui adhère fortement aux valeurs dites d'expression de soi (Inglehart et Welzel 2005). Ces dernières comportent de nombreuses similitudes avec les valeurs prônées par cette perspective. Ce parallèle confère donc à cette perspective un potentiel de résonnance intéressant auprès de la population masculine suisse.

Malgré ces caractéristiques, elle est très peu développée sur un plan politique, défendue par une seule organisation (Maenner.ch). En effet, les organisations qui partagent le constat d'une critique du rôle masculin traditionnel (Réseau hommes romand) suivent généralement un plan d'action basé sur la transformation identitaire et l'exploration de l'intime, et se distancient volontairement de toute forme d'action politique. De plus, la stratégie de recrutement, basée sur des réseaux informels, d'interconnaissances, représente, de facto, un fort obstacle à la mobilisation et à la diffusion des idées.

Enfin, comme les résultats de notre enquête auprès des membres le suggèrent, la perspective expressive et relationnelle est principalement soutenue par une élite d'hommes d'un niveau socioprofessionnel très élevé. Ainsi, l'identification de la majorité à une catégorie d'hommes privilégiés est susceptible de limiter la croissance du mouvement.

La perspective pro-féministe s'incarne principalement dans des ensembles très peu organisés, caractérisés par un très faible recrutement de membres et par une absence de ressources financières. Le constat du faible nombre d'hommes adhérant à cette perspective interroge. A certains égards, en effet, la perspective pro-féministe est en phase avec l'évolution de la société suisse contemporaine et avec un certain discours ambiant. On y relève, en effet, l'incitation adressée aux hommes à la réflexivité - voire à l'autocritique - à mieux se mettre à l'écoute et à participer aux tâches ménagères et de soin envers leur entourage familial, au même titre que les femmes, dans une perspective égalitariste. De plus, les hommes pro-féministes valorisent fortement l'horizontalité des rapports au sein du couple (et des rapports 
familiaux en général). Ces revendications font écho aux transformations récentes des rapports entre les sexes, en conformité aux éléments évoqués en introduction, abordant l'égalisation des statuts juridiques de l'homme et de la femme dans le couple. Toutefois, malgré l'actualité de ses propositions, la perspective pro-féministe rassemble très peu.

Cette difficulté à s'imposer pourrait provenir des défis qu'elle pose aux hommes souhaitant y adhérer. Premièrement, elle exige d'eux qu'ils reconnaissent, d'une part, l'existence de privilèges masculins et, d'autre part, leur participation au maintien et à la reproduction du système d'oppression des femmes. Peu d'hommes se sont retrouvés dans cette perspective, tout comme des femmes, plutôt associée au féminisme radical (Dulac 1994). Cette adhésion idéologique doit ensuite conduire à une mise en application pratique de ces principes. L'existence de groupes de paroles (Les Mâles barrés) était précisément conçue dans le but de déconstruire, par l'échange, cette part de masculinité intériorisée et reproductrice de la domination masculine. Par ailleurs, certains programmes allaient jusqu’à proposer aux hommes de s'engager à un renoncement effectif à leurs privilèges dans l'emploi, à travers une diminution substantielle de leur temps de travail (Hausmaennernetz).

Ainsi, la perspective pro-féministe, principalement tournée vers l'amélioration de la condition féminine, non seulement demande aux hommes d'admettre leur part de responsabilité et leur participation à ce système d'oppression, mais également de travailler à une amélioration de cette situation, par un renoncement effectif aux privilèges acquis.

Plusieurs travaux s'intéressant aux facteurs de mobilisation soulignent l'importance de réunir un certain nombre de conditions, afin de faciliter l'adhésion à une cause. Parmi celles-ci, Gamson (1992) décrit le rôle central du cadrage identitaire (identity frame) comme facteur de mobilisation. Il montre ainsi l'importance conjointe, à la fois du sentiment d'injustice (injustice frame) et d'identification forte (identity frame) avec le groupe qui se mobilise. Or, la perspective pro-féministe crée une situation paradoxale pour les hommes à cet égard. En effet, ces derniers sont portés à attribuer la responsabilité de l'injustice au groupe auquel ils sont assimilés (les hommes) et à s'identifier à un groupe mobilisateur auquel ils n'appartiennent pas (les femmes féministes). Ce paradoxe est, tout au moins, l'une des pistes qui peut être avancée pour expliquer le faible taux d'adhésion à cette perspective.

\section{Conclusion}

Cette recherche indique que les organisations masculines constituent un ensemble hétérogène sur le plan des perspectives sur la masculinité qu'elles prônent. Leurs orientations s'inscrivent dans des tendances sociétales globales. L'évolution des rapports hommes-femmes et des tendances sociales qui redéfinissent leurs rôles va 
de pair avec plusieurs remises en questions et besoins, auxquels les organisations masculines tentent de répondre. En effet, nous avons établi des parallèles entre des évolutions structurelles de la société helvétique et les modèles masculins qui semblent les accompagner. Ces résultats tendraient à confirmer l'hypothèse selon laquelle les changements dans les modèles culturels de genre rencontrent des évolutions conjointes aux reconfigurations structurelles (systèmes de lois, ensemble de pratiques) au sein d'une société donnée.

De plus, la présence des trois perspectives documentées dans cet article met en évidence des éléments de permanence du phénomène étudié, par-delà les contextes. En effet, au vu des résultats émergeant dans d'autres sociétés occidentales sur la même question, l'analyse des réactions collectives des hommes au processus de réalisation de l'égalité des sexes se traduit par des tensions similaires entre soutien (proféministes) versus résistances (droits des hommes, défense des droits des pères) à l'émancipation des femmes. En outre, l'affirmation d'une nouvelle masculinité libérée du joug d'un modèle de masculinité traditionnelle (mouvement de libération des hommes) révèle un autre front idéologique de ce mouvement.

Dans le contexte particulier de la Suisse, les résultats de notre étude permettent d'enrichir l'interprétation du phénomène d'émergence d'organisations masculines. Ils apportent, certes, une confirmation de la présence d'un ressac antiféministe en son sein. Toutefois, ce mouvement révèle également une adaptation à l'évolution récente des rapports entre les sexes, voire une forme de veille attentive à la situation des hommes dans le processus de marche vers l'égalité. Il n'est cependant pas possible de généraliser ces résultats à l'ensemble de la population masculine suisse, travail qu'il reste à mener.

Les résultats présentés dans cet article permettent également de constater la juxtaposition synchronique de modèles masculins diversifiés, voire opposés. Cela suggère que les modèles culturels de masculinité et de paternité, bien qu'étant le reflet d'évolutions historiques spécifiques, loin de se succéder, semblent se juxtaposer, se superposer au sein de la société suisse. Cependant il est difficile, à ce stade, de conclure à la prédominance de l'une ou l'autre des tendances examinées au sein de l'ensemble des organisations étudiées.

\section{Références bibliographiques}

Ben Salah, Hakim. 2013. Des hommes en mouvement. Vers une reconfiguration des modèles masculins? L'exemple de la Suisse. Thèse de doctorat, sciences sociales, Université de Lausanne.

BIT (Bureau international du travail). n. d. Classification internationale type des professions (CITP-88). Grands groupes, sous-grands groupes et sous-groupes. Laborsta Internet. Genève: BIT, http:// laborsta.ilo.org/applv8/data/isco88f.html (07.03.2013).

Blais Mélissa et Francis Dupuis-Déri. 2008. Le mouvement masculiniste au Québec. L'antiféminisme démasqué. Montréal : Remue-Ménage. 
Boys to Men. 2009. http://boystomen.ch/joomla/ (04.08.2009).

Bunge, Mario. 2003. Philosophical dictionary. New York: Prometheus Books.

Carnal, Mathieu. 2006. La montée des mouvements d'hommes en Suisse? Que veut vraiment Maenner. ch? L'Emilie 1499: 20-21.

Clatterbaugh, Kenneth. 1997. Contemporary perspectives on masculinity. $2^{\text {nd }}$ edition. Boulder: Westview Press.

Corneau, Guy. 1989. Père manquant, fils manqué. Montréal: Editions de l'Homme.

CROP (Coordination romande des organisations paternelles). 2009a. Maltraitances d'enfants par leurs parents. CROP, http://www.crop.ch/images/coordination/pdf/20090318_PositionCrop-Annexe1. $\operatorname{pdf}(08.10 .2009)$.

CROP (Coordination romande des organisations paternelles). 2010b. Prise de position de la CROP concernant le projet de révision du code civil et du code pénal. CROP, http://www.crop.ch/ images/coordination/pdf/20090318_PositionCrop.pdf (09.10.2009).

DFJP (Département fédéral de justice et police). 2009. Documentation - Autorité parentale. Rapport relatif à la révision du code civil (autorité parentale) et du code pénal (art. 220). Berne: Office fédéral de la justice, http://www.ejpd.admin.ch/content/dam/data/gesellschaft/gesetzgebung/ elterlichesorge/vn-ber-f.pdf(19.03.2013).

Dufresne, Martin. 1998. Masculinisme et criminalité sexiste. Recherches féministes 11(2) : 125-137.

Dulac, Germain. 1994. Penser le masculin: essai sur la trajectoire des militants de la condition masculine et paternelle. Québec: Institut québécois de recherche sur la culture.

Dupuis-Déri, Francis. 2004. Féminisme au masculin et contre-attaque "masculiniste» au Québec. Mouvements 31(1): 70-74.

Flood, Michael, Judith K. Gardiner, Bob Pease et Keith Pringle (éds.). 2007. International encyclopedia of men and masculinities. New York: Routledge.

Gamson, William A. 1992. Talking politics. Cambridge: Cambridge University Press.

IGM Schweiz. 2009. Lohndiskriminierung - Das Märchen von den 25\%. Aarau : IGM Schweiz, http:// www.igm.ch/es-war-einmal/ (23.11.2009).

Inglehart, Ronald et Christian Welzel. 2005. Modernization, cultural change, and democracy: the human development sequence. New York: Cambridge University Press.

Karoski, Spase. 2007. Men on the move: the politics of the men's movement. Thèse de doctorat, University of Wollongong, Australie.

Kimmel, Michael S. et Michael A. Messner. 1989. Introduction. Pp 1-13 in Men's lives, édité par Michael S. Kimmel et Michael A. Messner. New York: Macmillan.

Lindsay, Jocelyn, Gilles Rondeau et Jean-Yves Desgagnés. 2011. Bilan et perspectives du mouvement social des hommes au Québec entre 1975 et 2010. Pp. 13-43 in Regards sur les hommes et les masculinités. Comprendre et intervenir, édité par Jean-Martin Deslauriers, Gilles Tremblay, Sacha Genest-Dufault, Daniel Blanchette et Jean-Yves Desgagnés. Québec: Presses de l'Université Laval.

Lingard Bob et Peter Douglas. 1999. Men Engaging Feminisms. Pro-feminism, Backlashes and Schooling. Philadelphia: Open University Press.

Macho Man. 2009. http://www.macho-man.ch (17.07.2009).

Malik, Khalid (dir.). 2013. Rapport sur le développement humain 2013. New York: Programme des nations unies pour le développement.

Messner, Michael. 1997. Politics of Masculinities. Lanham: Alatamira Press.

Miller, Frederick D. 1999. The end of SDS and the emergence of weatherman: demise through success. Pp. 303-324 in Waves of Protest: Social Movements since The Sixties, édité par Jo Freeman et Victoria Johnson. Lanham: Rowman \& Littlefield Publishers.

Mucchielli, Roger. 2006. L'analyse de contenu. Des documents et des communications. Paris: ESF éditeur. 
OFS (Office fédéral de la statistique). 2014a. Enquête - Enquête suisse sur la structure des salaires. Fiche signalétique. Neuchâtel: OFS, http://www.bfs.admin.ch/bfs/portal/fr/index/regionen/thematische_karten/gleichstellungsatlas/erwerbsarbeit_und_beruf/lohnungleichheit.html (25.07.2014).

OFS (Office fédéral de la statistique). 2014b. Enquête - Enquête suisse sur la population active (ESPA). Fiche signalétique. Neuchâtel: OFS, http://www.bfs.admin.ch/bfs/portal/fr/index/themen/01/04/ blank/01/04/01.html (22.07.2014).

OFS (Office fédéral de la statistique). 2014c. Source - Statistique des élections. Fiche signalétique. Neuchâtel: OFS, http://www.bfs.admin.ch/bfs/portal/fr/index/themen/17/02/blank/key/ frauen_und_politik/bund.html (25.07.2014).

OFS (Office fédéral de la statistique). 2014d. Enquêtes - SIUS, ESPOP, STATPOP. Fiche signalétique. Neuchâtel: OFS, http://www.bfs.admin.ch/bfs/portal/fr/index/themen/20/05/blank/key/ gleichstellung_und/abschlussquoten.html (25.07.2014).

OFS (Office fédéral de la statistique). 2014e. Enquêtes - BEVNAT, ESPOP, STATPOP. Fiche signalétique. Neuchâtel: OFS, http://www.bfs.admin.ch/bfs/portal/fr/index/themen/01/06/blank/ key/06.html (25.07.2014).

OFS (Office fédéral de la statistique). 2014f. Enquête - BEVNAT. Fiche signalétique. Neuchâtel: OFS, http://www.bfs.admin.ch/bfs/portal/fr/index/themen/01/06/blank/data/03.html

(12.07.2014).

Réseau hommes romand. 2009. Accueil. Réseau hommes romand, http://www.rhsr.com (12.08.2009).

Reusser, Ruth. 2006. Divorce. Dictionnaire historique de la Suisse, http://www.hls-dhs-dss.ch/textes/f/ F7993.php?topdf $=1$ (15.2.2013).

Schiess, Christian. 2007. Masculinistes contre féminisme. L'Emilie 1507: 12-13.

Theunert, Markus. 2012. Männerpolitik in der Schweiz. Pp. 423-445 in Männerpolitik. Was Jungen, Männer und Väter stark macht, édité par Markus Theunert. Wiesbaden: Springer.

Thiers-Vidal, Léo. 2002. De la masculinité à l'anti-masculinisme: penser les rapports sociaux de sexe à partir d'une position sociale oppressive. Nouvelles Questions Féministes 21(3) : 71-83.

Welzer-Lang, Daniel. 2011. Epistémologie des études critiques sur les hommes et le masculin. Point de vue situé d'un garçon de France, après 25 ans de recherches sur ces thèmes. Contribution présentée au Colloque international Perspectives futures en intervention, politique et recherche sur les hommes et les masculinités. Laval, Québec, 11 mars 2011. 